\title{
Per un dibattito sul management nell'era digitale Riflessioni sul libro: Francesco Varanini, Le cinque leggi bronzee dell'era digitale $e$ perché conviene trasgredirle, Guerini e Associati, Milano, 2020 \\ by Claudio Baccarani
}

Con questo testo Francesco Varanini si propone di "raccontare fasti e nefasti dell'Era Digitale" (p. 15). Lo fa ricorrendo ad un linguaggio tra il tecnico e il divulgativo, mosso dal desiderio di aprire un dialogo sugli innumerevoli e preziosi vantaggi dei processi di digitalizzazione, alla luce dei rischi che ne potrebbero conseguire.

Rischi derivanti dall' evoluzione della ricerca sulle tecnologie digitali verso forme di Intelligenza Artificiale, che potrebbero dotare le macchine di pensiero con la possibilità di sostituire completamente le persone sul posto di lavoro e di porre le basi di una società dai contorni non certo ben definiti, ma che vedrebbe la compresenza di esseri umani e di altre creature, quali ad esempio androidi, il cui nome, guarda caso, è stato adottato dal sistema operativo di Google (android).

Situazione del tutto nuova nella storia dell'umanità, che ha sempre visto la tecnologia supportare e trasformare le varie forme di lavoro anche attraverso sconvolgimenti come quelli occorsi a seguito della prima rivoluzione industriale - ma mai prospettare una generalizzata fuoriuscita dal lavoro delle persone, sostituite da macchine capaci di pensare, con la conseguente necessità di rivedere alla base la configurazione del sistema sociale di riferimento.

Confesso che a temi di questo tipo non ho mai dedicato tanta attenzione, se non in gioventù, nelle mie vesti di appassionato lettore di romanzi di fantascienza pubblicati nella storica collana Urania, edita da Mondadori a partire dal 1952, o come spettatore di film sulla stessa lunghezza d'onda, tra i quali subito la memoria corre a "2001 Odissea nello spazio" di Stanley Kubrick (1968), ad “A.I. Artificial Intelligence” (2001) di Steven Spielberg e a "Minority Report" (2002) dello stesso autore.

Mi sono quindi avvicinato con circospezione e prudenza alla lettura del testo, spinto da una risoluta curiosità e dal desiderio di esplorare mondi in gran parte nuovi alla mia esperienza.

Il libro si apre con una premessa apparentemente lontana dal tema peraltro opportunamente senza titolo - nella quale si accostano pensiero letterario (Leopardi e Goethe) e pensiero politico-economico (Lassalle e Marx) nella condivisione dell'esistenza di leggi bronzee, cioè, solide, durevoli e resistenti che caratterizzano il divenire delle diverse epoche storiche.

La sorpresa generata da questa apertura ha stimolato ancor più la mia curiosità. Così, sono stato catturato dalla lettura dei capitoli successivi condotti con una narrazione ritmata e incalzante, caratterizzata da frequenti 
sinergie italian journal of management

rimandi e richiami che ne fanno risaltare il carattere di costruzione letteraria romanzesca, il cui profilo potrebbe ben proporsi come base per la sceneggiatura di una rappresentazione teatrale o di un film.

La costruzione dell'A. appare, infatti, come un'inchiesta che narra in una forma brillante, cadenzata e del tutto originale il divenire dei progressi in campo digitale. Lo fa attraverso il racconto delle scelte di esperti che hanno lavorato, e lavorano, su queste tecnologie, scandagliandone in profondità le posizioni e la visione nel contesto del loro vissuto personale.

In questo modo prendono forma le cinque leggi bronzee che campeggiano nei titoli dei capitoli centrali del libro: "ti arrenderai a un codice straniero", "preferirai la macchina a te stesso", "non sarai più cittadino: sarai suddito o tecnico", "lascerai alla macchina il governo", "vorrai essere macchina". Capitoli dedicati a porre in risalto in modo dettagliato e circostanziato i possibili impatti della citata evoluzione tecnologica sulla vita degli individui, che gradualmente potrebbero divenire parte di una massa indistinta dominata da una tecnocrazia composta da una ristretta cerchia di potenti tecnici e costruttori.

Il sottotitolo del libro invita però a trasgredire queste leggi, per riaffermare il tratto umano della persona nellagire per il bene, il bello e il buono come elemento differenziale rispetto a macchine che, seppur progredite, sono chiamate ad agire sotto il suo controllo.

Invito che prende forma nel capitolo finale attraverso i verbi che ne scolpiscono il titolo, "distinguere, giudicare, scegliere", e l'approccio letterario, filosofico e sociologico che lo caratterizza nel ribadire la centralità della persona per le scelte fondanti la costruzione del futuro.

Emblematici al riguardo sono due passaggi del testo nei quali vengono descritte le differenze tra Homo digitalis e Homo sapiens e viene proposta una creativa narrazione della funzione del computer attraverso l'immedesimazione dell'A. con Nestore il grande narratore del mondo omerico.

Nel primo dei due passaggi citati Varanini chiarisce che: "l'Homo digitalis riduce il suo sforzo di conoscere al porre domande alla macchina: si fida di un algoritmo. Conosce e si relaziona con gli altri essere umani attraverso macchine e algoritmo (p. 274)......mentre......l'Homo sapiens sa che esistono varie maniere per conoscere il mondo, se stesso e gli altri. Usare le macchine è solo uno dei modi possibili, non il migliore. ...Per cautela, non fa tutto ciò che la macchina chiede; ricorda sempre che non gli è dato di sapere cosa è scritto nel codice; si protegge per quanto possibile dall'incessante osservazione della macchina" (p. 275).

Così l'Homo digitalis fugge dalla complessità delle relazioni umane $\mathrm{e}$ opta per un isolamento individuale nella semplicità di un mondo vissuto attraverso le macchine. Al contrario, l'Homo sapiens accetta la complessità del mondo reale, all'interno del quale si pone alla continua ricerca di senso per quello che come persona e individuo è chiamato a fare nella comunità in cui vive attraverso la ricerca, la narrazione, le relazioni umane, la cultura e l'arte come esaltazione della propria capacità inventiva ed espressiva.

$\mathrm{Nel}$ secondo dei due passi citati l'A. immagina, invece, un ideale racconto di Nestore nel quale ogni persona è come un viandante in cammino attraverso un presente che lo conduce "dal passato al futuro, dal 
noto all'ignoto". In questo incessante viaggio è supportata da un bastone, il computer, "al quale appoggiarsi, al quale ricorrere per aumentare il raggio e la potenza della propria azione"; da una bisaccia, il computer, "lo strumento leggero che può essere portato sempre con sé...che permette di mantenere a disposizione gli utensili" necessari al viaggio; da una scarpa vecchia, il computer,....... lo strumento plastico che si conforma al corpo, al pensiero, alle intenzioni, alle necessità della persona che l'ha in uso" (292-293).

In questo modo, viene posto in risalto il valore di uno strumento progredito che può accompagnare e sostenere la persona nel proprio "viaggio nell'esplorazione del mondo", in un cammino plasticamente e metaforicamente rivelato da Charlie Chaplin nel finale di Tempi Moderni, quando Charlot e la Monella, provati, ma mai sconfitti, si incamminano mano nella mano sulla strada dell'avvenire. Tutto questo però a patto che si sappia resistere al "soave incantamento" di macchine che reclamano attenzione come le sirene di Ulisse.

Con questo scritto l'A. si propone di lanciare un allarme alla società civile che inconsapevolmente viaggia nella direzione descritta per almeno due motivi. Da una parte, la condizione di disinformazione, o di incredulità, nella quale versa il cittadino al riguardo di un fenomeno costruito con linguaggi e codici inaccessibili ai non tecnici e in gran parte mantenuto circoscritto al loro ristretto ambito di studio e discussione. Dall'altra, il muovere strisciante verso questo orizzonte secondo forme impalpabili dal "fascino discreto", che abituano gradualmente le persone alla dipendenza dalle macchine, come ognuno di noi può sperimentare nel proprio vivere quotidiano, se si ferma un momento ad osservare il divenire del rapporto con le "macchine digitali" che usa.

Riconoscendo il valore dei progressi delle tecnologie digitali, il libro non si muove sui piani della rassicurazione o del catastrofismo, traccia, piuttosto, dei percorsi e lascia trasparire tutta una serie di domande rispetto alle quali il Lettore è chiamato a prendere posizione.

In realtà, numerosi sono gli interrogativi generati dalla lettura del libro: è mai possibile che le macchine prendano il controllo delle persone? qual è il confine tra realtà e fantascienza? perché di questi temi pur se con intensità crescente non si parla che a livello di addetti ai lavori? discutere di questi temi significa porsi contro il progresso scientifico? abbiamo mai riflettuto su tutto questo nell'ambito del nostro vissuto quotidiano? perché gli studi condotti sull'argomento frequentemente non introducono riferimenti storici? perché gli aspetti etico-filosofici di questa possibile evoluzione sono posti in secondo piano? è possibile capire il presente e il futuro di questa condizione senza una conoscenza del passato e l'intelligenza poetico- letteraria di leggere tra le righe del cambiamento?

Ma soprattutto, cosa accadrà nel mondo del lavoro e delle relazioni sociali a seguito di una più o meno intensa riduzione di posti di lavoro a seconda del livello di sviluppo dei diversi Paesi? Non è che questa robotizzazione intelligente possa porsi come un "cigno nero" imprevedibile e incontrollabile nei suoi effetti sociali ben più di una pandemia? Non siamo di fronte ad un nuovo paradigma che ci obbliga a ripensare radicalmente e a reinventare il management nel governo dell'impresa e delle relazioni 
sinergie

Vol. 39, Issue 1, 202

organizzative e sociali? (Jacques Martin, 2017).

E ancora, come si dovrà distribuire la ricchezza prodotta nelle fabbriche, magari a luci spente, come le lights-out factoris di Philip Dick (p. 149) dove il buio domina perché popolate solo da macchine che non necessitano della luce? Per cosa saranno remunerate le persone che perdono il loro lavoro? Come dovranno essere ridefinite le relazioni tra stato e imprese dal punto di vista delle relazioni sociali? Quali saranno i nuovi spazi di lavoro? Perché questo tema non è così evidente nelle discussioni delle parti sociali? È possibile un'epoca della felicità senza lavoro (Primo Levi, 1978)?

Queste solo alcune domande. Di certo ogni Lettore ne troverà altre alle quali sarà eticamente chiamato dal proprio Sé a cercare una risposta.

E questa capacità del libro di generare domande costituisce un significativo contributo del lavoro dell'A., per la forza che le domande possiedono come sostegno ai processi di innovazione e cambiamento (Jostein Gaarder, 1997).

Contributo che scaturisce dal valore delle approfondite ricerche condotte, che traspare anche dalla copiosa bibliografia curata secondo un ormai inusuale, quanto prezioso, indice analitico, oltre che dalla puntigliosa indicazione dei periodi di consultazione dei materiali su internet. Ma anche dalla capacità dell'A. di catturare l'attenzione del Lettore coinvolgendolo nella narrazione.

Come detto, ho letto il libro di Francesco Varanini a digiuno del tema trattato, quindi da semplice componente di quella società civile che nelle prospettive delineate rischia la trasformazione in sudditanza o addirittura in schiavitù delle macchine.

Per questo sono ancor più grato all'A. perché con questo suo lavoro apre una breccia nel pesante "silenzio pubblico" sul tema e traccia un percorso a sostegno di scelte capaci di condurre la società civile a godere dei vantaggi dell'Intelligenza Artificiale attraverso un "essere umani tramite la tecnica" (p. 276).

Lo fa consapevole della forza e del valore della memoria storica, dell'arte e della bellezza che non possono essere immagazzinate in una macchina, perché sempre rigenerate in un continuo movimento con le esperienze dei singoli individui.

Così, condividendo questa prospettiva di approfondimento, mi auguro che il tema possa uscire dalle ristrette analisi e valutazioni degli esperti per poter coinvolgere in un approccio interdisciplinare e pubblico le voci in grado di disegnare le tappe di un progredire sociale comune.

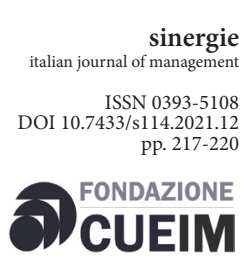

S I

$M A$

Italian Society of MANAGEMENT

\section{Claudio Baccarani}

Emerito di Economia e Gestione dell'Impresa

Università di Verona

e-mail: claudio.baccarani@univr.it

\section{Riferimenti bibliografici}

GAARDER J. (1997), Cè nessuno?, Salani Editore, Firenze.

LEVI P. (1978), La chiave a stella, Einaudi, Torino.

MARTIN J. (2017), "Who's afraid of Virginia Woolf", Sinergie italian journal of management, vol. 35, n. 103, pp. 241-252. 